\title{
Thermal wave scattering by two overlapping and parallel cylinders
}

\author{
R. Celorrio • M. Costa • A. Mendioroz • E. Apiñaniz • \\ S.M. Shibli • A. Salazar
}

Received: 20 May 2008 / Accepted: 17 July 2008 / Published online: 7 August 2008

(C) Springer-Verlag 2008

\begin{abstract}
In this paper an analytical solution of the temperature of an opaque material containing two overlapping and parallel subsurface cylinders, illuminated by a modulated light beam, is presented. The method is based on the expansion of plane and cylindrical thermal waves in series of Bessel and Hankel functions. This model is addressed to the study of heat propagation in composite materials with interconnection between inclusions, as is the case of inverse opals and fiber reinforced composites. Measurements on calibrated samples using lock-in infrared thermography confirm the validity of the model.
\end{abstract}

PACS 66.70.+f $\cdot$ 81.70.Fy $\cdot 44.10 .+\mathrm{I} \cdot 66.30 . \mathrm{Xj}$

\section{Introduction}

Photothermal techniques are very powerful tools for the thermophysical characterization and nondestructive evaluation (NDE) of a wide variety of materials [1]. They are based on the generation and detection of thermal waves in

R. Celorrio - M. Costa

Departamento de Matemática Aplicada, EUITIZ, Universidad de Zaragoza, Campus Río Ebro, Edificio Torres Quevedo, 50018

Zaragoza, Spain

M. Costa $\cdot$ S.M. Shibli

Instituto de Física, Universidade de São Paulo, Caixa Postal 66318, 05315-970, São Paulo, SP, Brazil

A. Mendioroz $\cdot$ E. Apiñaniz $\cdot$ A. Salazar $(\varangle)$

Departamento de Física Aplicada I, Escuela Técnica Superior de Ingeniería, Universidad del País Vasco, Alameda Urquijo s/n, 48013 Bilbao, Spain

e-mail: agustin.salazar@ehu.es the sample under study. In the case of modulated techniques, thermal waves are generated as a consequence of the absorption of an intensity modulated light beam. These highly damped thermal waves propagate through the material and are scattered by the subsurface heterogeneities. Different photothermal setups have been developed to detect these thermal waves and therefore to extract information on the thermal properties and internal structure of the material: Infrared radiometry, mirage effect, photothermal reflectance, etc. [1].

In the last years several studies on the propagation of thermal waves in materials with plane [2,3], cylindrical $[4,5]$ and spherical $[6]$ inclusions have been published. The results of these works show that photothermal techniques are suitable to study the thermal properties of multilayer samples, fiber reinforced composites, and particulate reinforced composites, respectively. In these studies it is assumed that there is no interconnection between inclusions. However, in some real materials with periodic arrangements of cylindrical or spherical particles, a certain degree of connection between them actually exists. Such is the case of inverse opals, which consist of a regular arrangement of spherical overlapping voids surrounded by solid walls [7]. On the other hand, in the case of composites with a high fiber's volume fraction, unexpected high values of the effective thermal conductivity have been found [8]. This is probably due to a direct contact between fibers, favoring heat propagation directly through the highly thermal conducting fibers. This contact reduces the high thermal resistance resulting from the in-series stack of highly conducting fiber-poor conducting matrix. Therefore to study their thermal properties we need to know how heat propagates through these connected arrays of cylinders and spheres.

The aim of this paper is to study the multiple scattering of thermal waves by two overlapping cylinders. In this way, an 
analytical solution of the temperature of an opaque material, illuminated by a modulated light beam, with two overlapping parallel cylinders buried beneath the surface has been found. We have used a method based on the expansion of plane and cylindrical thermal waves in series of Bessel and Hankel functions. We have performed measurements on calibrated samples using lock-in infrared thermography which confirm the validity of the model.

\section{Theory}

To introduce the theoretical model we first study the case of two parallel and infinite nonoverlapping buried cylinders. Then we will include the modifications to the previous model due to the overlapping of the cylinders. In the following the material is assumed to be semi-infinite and opaque, and its surface is illuminated by a plane light beam modulated at a frequency $f(\omega=2 \pi f)$. Hereafter, subscripts $M$ and $C$ stand for matrix and cylinders, respectively.

\subsection{Non-overlapping cylinders}

The geometry of the problem is shown in Fig. 1(a). In the absence of any subsurface heterogeneity the temperature is given by [1]:

$T(x, t)=T_{o} e^{-i\left[q_{M}(x-d)+\omega t\right]}$,

where $T_{o}$ is a function of the light intensity and of the thermal properties of the matrix, $q=(1+i) / \mu$ is the thermal wave vector, $\mu=(D / \pi f)^{0.5}$ being the thermal diffusion length and $D$ the thermal diffusivity. Equation (1) represents a highly damped plane thermal wave generated at the sample surface $(x=d)$ and propagating along $x<0$.

In the problem we are dealing with, two infinite and parallel cylinders of radius $a_{1}$ and $a_{2}$, are buried at depths $d_{1}$ and $d_{2}$, respectively. The horizontal distance between centers is $h$. The sample surface is surrounded by air whose thermal conductivity is negligible. Due to this adiabatic boundary condition at the sample surface $(\nabla T=0)$, the real geometry shown in Fig. 1(a) can be analyzed by considering the configuration shown in Fig. 1(b). The effect of the wall is accounted for via the introduction of two image cylinders and an image plane thermal wave.

Due to the linear nature of the heat diffusion equation, the spatial component of the temperature at any point of the material $\left(T_{M}\right)$ can be written as the superposition of the incident plane thermal wave $\left(T_{\mathrm{inc} 1}\right)$ with the scattered waves coming out from the two real cylinders and from their images:

$T_{M}\left(r_{1}, \theta_{1}\right)=T_{\text {inc1 }}\left(r_{1}, \theta_{1}\right)+\sum_{j=1}^{4} T_{\text {scatt }}^{(j)}\left(r_{1}, \theta_{1}\right)$.

The incident plane thermal wave can be expanded in series of cylindrical waves by using the Bessel functions of the first kind $\left(J_{m}\right)[9]$ :

$$
\begin{aligned}
T_{\mathrm{inc} 1}\left(r_{1}, \theta_{1}\right) & =T_{o} e^{-i q_{M}\left(x_{1}-d_{1}\right)} \\
& =T_{o} e^{i q_{M} d_{1}} \sum_{m=-\infty}^{\infty} i^{-m} J_{m}\left(q_{M} r_{1}\right) e^{i m \theta_{1}}
\end{aligned}
$$

while the scattered cylindrical waves can be expanded in series of Hankel functions of the first kind $\left(H_{m}\right)$ [10]:

$$
\begin{aligned}
& T_{\text {scatt }}^{(j)}\left(r_{j}, \theta_{j}\right)=\sum_{m=-\infty}^{\infty} A_{m}^{(j)} H_{m}\left(q_{M} r_{j}\right) e^{i m \theta_{j}}, \\
& j=1, \ldots, 4 .
\end{aligned}
$$

Note that each scattered wave is referred to a coordinate frame centered in the own cylinder. However, by using Graf's addition theorem, the scattered waves emerging
Fig. 1 (a) Geometry used to study the scattering of plane thermal waves by two subsurface cylinders.

(b) Equivalent geometry after applying the image theorem
Plane illumination

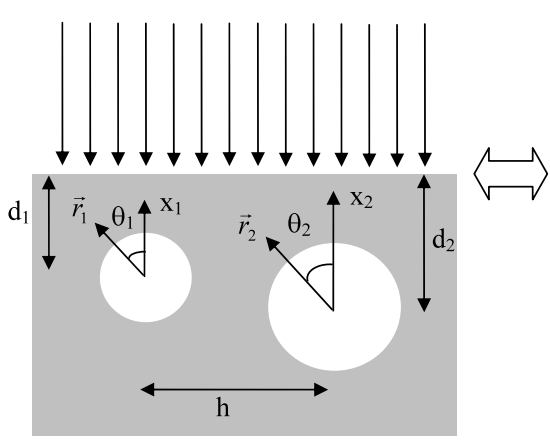

$\mathbf{a}$

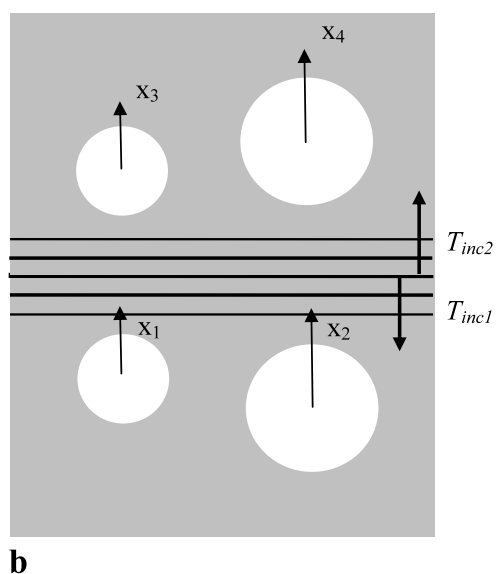

b 
Fig. 2 Geometry for Graf's theorem

from one cylinder can be expressed in the coordinate frame of another one (see Fig. 2) [11]:

$X_{m}\left(r_{2}\right) e^{i m \theta_{2}}=\sum_{n=-\infty}^{\infty} X_{n-m}\left(r_{12}\right) J_{n}\left(r_{1}\right) e^{i n \theta_{1}} e^{-i(n-m) \theta_{12}}$

where $X_{m}$ means Bessel or Hankel functions, but for the latter, (5) is restricted to $r_{1}<r_{12}$. Accordingly, the matrix temperature referred to the coordinates centered in each cylinder is given by:

$T_{M}\left(r_{j}, \theta_{j}\right)=\sum_{m=-\infty}^{\infty} \lambda_{m}\left(r_{j}\right) e^{i m \theta_{j}}, \quad j=1,2$,

where the coefficients $\lambda_{m}\left(r_{j}\right)$ are:

$$
\begin{aligned}
\lambda_{m}\left(r_{1}\right)= & T_{o} e^{i q_{M} d_{1}} i^{-m} J_{m}\left(q_{M} r_{1}\right)+A_{m}^{(1)} H_{m}\left(q_{M} r_{1}\right) \\
& +\sum_{n=-\infty}^{\infty} A_{n}^{(2)} H_{m-n}\left(q_{M} r_{12}\right) J_{m}\left(q_{M} r_{1}\right) e^{-i(m-n) \theta_{12}} \\
& +\sum_{n=-\infty}^{\infty} A_{-n}^{(1)} H_{m-n}\left(2 q_{M} d_{1}\right) J_{m}\left(q_{M} r_{1}\right) \\
& +\sum_{n=-\infty}^{\infty} A_{-n}^{(2)} H_{m-n}\left(q_{M} r_{14}\right) J_{m}\left(q_{M} r_{1}\right) e^{-i(m-n) \theta_{14}},
\end{aligned}
$$

$$
\begin{aligned}
\lambda_{m}\left(r_{2}\right)= & T_{o} e^{i q_{M} d_{2}} i^{-m} J_{m}\left(q_{M} r_{2}\right) \\
& +\sum_{n=-\infty}^{\infty} A_{n}^{(1)} H_{m-n}\left(q_{M} r_{21}\right) J_{m}\left(q_{M} r_{2}\right) e^{-i(m-n) \theta_{21}} \\
& +A_{m}^{(2)} H_{m}\left(q_{M} r_{2}\right) \\
& +\sum_{n=-\infty}^{\infty} A_{-n}^{(1)} H_{m-n}\left(q_{M} r_{23}\right) J_{m}\left(q_{M} r_{2}\right) e^{-i(m-n) \theta_{23}} \\
& +\sum_{n=-\infty}^{\infty} A_{-n}^{(2)} H_{m-n}\left(2 q_{M} d_{2}\right) J_{m}\left(q_{M} r_{2}\right) .
\end{aligned}
$$

Here we have used the relations between the constants: $A_{m}^{(3)}=A_{-m}^{(1)}$ and $A_{m}^{(4)}=A_{-m}^{(2)}$, which follow from the symmetry condition between each cylinder and its image: $T_{\text {scatt }}^{(1)}(r, \theta)=T_{\text {scatt }}^{(3)}(r, \pi-\theta)$ and $T_{\text {scatt }}^{(2)}(r, \theta)=T_{\text {scatt }}^{(4)}(r, \pi-\theta)$.

On the other hand, the temperature inside the cylinders can be expanded in terms of the ordinary Bessel functions [12]:

$T_{c}^{(j)}\left(r_{j}, \theta_{j}\right)=\sum_{m=-\infty}^{\infty} B_{m}^{(j)} J_{m}\left(q_{c} r_{j}\right) e^{i m \theta_{j}}, \quad j=1,2$.

The solution of the temperature field in the material and in the two cylinders requires the knowledge of the value of the $4 m$ constants $A_{m}^{(1)}, A_{m}^{(2)}, D_{m}^{(1)}$, and $D_{m}^{(2)}$. They can be obtained from the boundary conditions at the cylinders surfaces:

(a) Temperature continuity:

$$
T_{M}\left(r_{j}=a_{j}\right)=T_{c}^{(j)}\left(r_{j}=a_{j}\right), \quad j=1,2 .
$$

(b) Heat flux continuity:

$$
K_{M} \nabla T_{M}\left(r_{j}=a_{j}\right)=K_{c} \nabla T_{c}^{(j)}\left(r_{j}=a_{j}\right), \quad j=1,2 .
$$

Substituting (6) and (8) into (9), we obtain the following system of $4 m$ equations $(m=-\infty, \ldots, 0, \ldots,+\infty)$ :

$\lambda_{m}\left(a_{j}\right)=B_{m}^{(j)} J_{m}\left(q_{c} a_{j}\right), \quad j=1,2$,

$K_{M} \lambda_{m}^{\prime}\left(a_{j}\right)=K_{c} q_{c} B_{m}^{(j)} J_{m}^{\prime}\left(q_{c} a_{j}\right), \quad j=1,2$,

where $K$ is the thermal conductivity, and $\lambda_{m}^{\prime}$ and $J_{m}^{\prime}$ are the derivatives of the functions $\lambda_{m}$ and $J_{m}$. By substituting the obtained values of the constants $A_{m}^{(1)}, A_{m}^{(2)}, B_{m}^{(1)}$, and $B_{m}^{(2)}$ into (6) and (8), the temperature at any point of the matrix and cylinders is obtained.

It is worth noting that (10) can be expressed in a matrix form which allows one to incorporate an unlimited number of buried cylinders in an easy way [4]. On the other hand, the method is also valid for a periodic line illumination parallel to the cylinders, whose geometry is shown in Fig. 3. Equations (6), (8), and (10) can be used to calculate the temperature in the matrix and inside the cylinders, provided that the first term in the right-hand side of (7), which accounts for the contribution of the incident plane thermal wave, is changed by the corresponding expression for an incident cylindrical thermal wave [13]:

$$
\begin{aligned}
& T_{o} e^{i q_{M} d_{j}} i^{-m} J_{m}\left(q_{M} r_{j}\right) \\
& \quad \rightarrow T_{1} i H_{m}\left(i q_{M} r_{j 0}\right) J_{m}\left(q_{M} r_{j}\right) e^{i m \theta_{j}} e^{-i m \theta_{j 0}}, \\
& \quad j=1,2
\end{aligned}
$$

where $T_{1}$ is a function of the heating light power and of the thermal properties of the matrix. 


\subsection{Overlapping cylinders}

The geometry of the two overlapping and parallel cylinders is shown in Fig. 4. The angles we have used are described in Fig. 5. We restrict our calculations to the so-called "small overlapping" problem, i.e., the center of the cylinder cannot be inside the other one. This is due to the fact that the validity of Graf's theorem (5) for the Hankel functions is restricted to $r_{1}<r_{12}$.

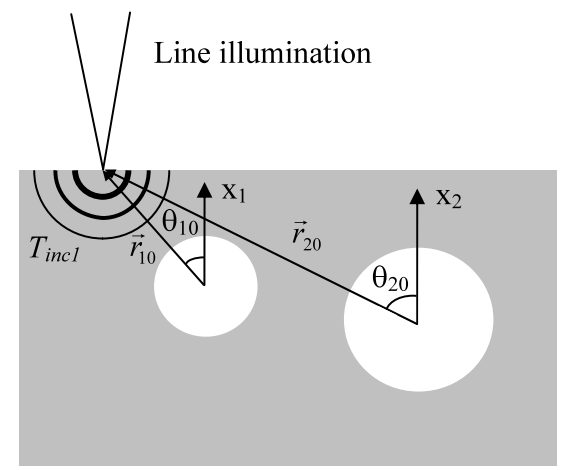

Fig. 3 The same geometry as in Fig. 1 but with line illumination parallel to the cylinders
Now we use Graf's theorem to transform (8) in order to express the temperature inside each cylinder as a function of the coordinates frame of the other one:

$T_{c}^{(i)}\left(r_{j}, \theta_{j}\right)=\sum_{m=-\infty}^{\infty} \eta_{m}^{(i)}\left(r_{j}\right) e^{i m \theta_{j}}, \quad i, j=1,2, i \neq j$,

where the functions $\eta_{m}^{(i)}\left(r_{j}\right)$ are:

$\eta_{m}^{(i)}\left(r_{j}\right)=\sum_{n=-\infty}^{\infty} B_{n}^{(i)} J_{m-n}\left(q_{c} r_{j i}\right) J_{m}\left(q_{c} r_{j}\right) e^{-i(m-n) \theta_{j i}}$

$i, j=1,2, i \neq j$.

Whereas (2) to (8) are still valid for overlapping cylinders, the boundary conditions for temperature and heat flow continuity now are:

$$
\begin{aligned}
T_{c}^{(j)} & \left(r_{j}=a_{j}\right) \\
& = \begin{cases}T_{M}\left(r_{j}=a_{j}\right) & \text { for } \theta_{j} \notin\left[\alpha_{j 1}, \alpha_{j 2}\right] \\
T_{c}^{(i)}\left(r_{j}=a_{j}\right) & \text { for } \theta_{j} \in\left[\alpha_{j 1}, \alpha_{j 2}\right]\end{cases} \\
& =\sum_{m=-\infty}^{\infty}\left[\lambda_{m}\left(a_{j}\right)+\gamma_{m}\left(a_{j}\right)\right] e^{i m \theta_{j}}, \quad i, j=1,2, i \neq j,
\end{aligned}
$$

Fig. 4 (a) Geometry used to study the scattering of plane thermal waves by two overlapped and parallel subsurface cylinders. (b) Equivalent geometry after applying the image theorem

Fig. 5 Angles used for two overlapping and parallel cylinders

Plane illumination

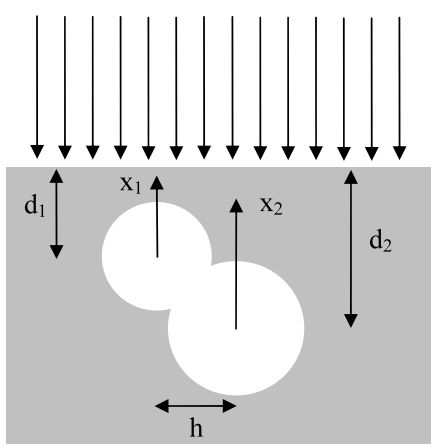

a

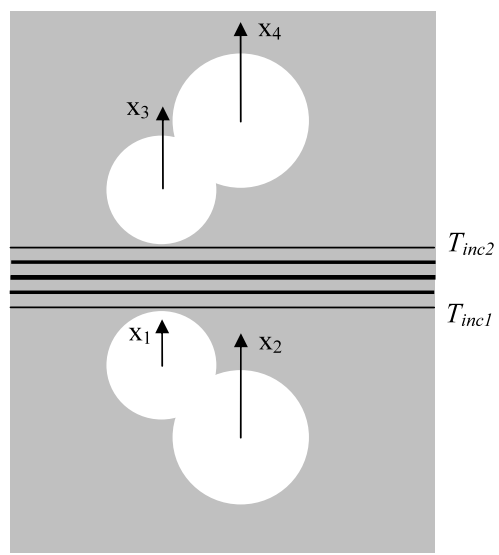

b
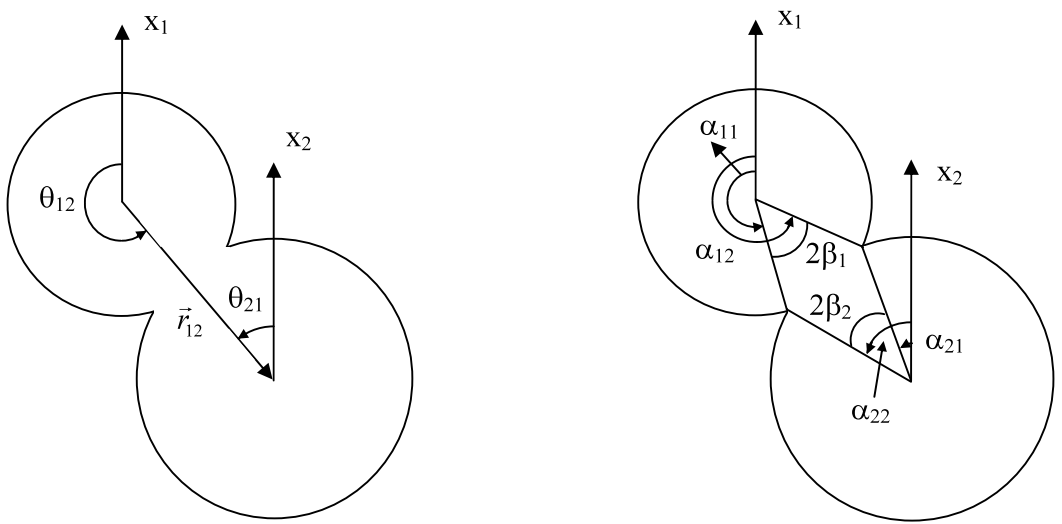


$$
\begin{aligned}
K_{c} & \nabla T_{c}^{(j)}\left(r_{j}=a_{j}\right) \\
& = \begin{cases}K_{M} \nabla T_{M}\left(r_{j}=a_{j}\right) & \text { for } \theta_{j} \notin\left[\alpha_{j 1}, \alpha_{j 2}\right] \\
K_{c} \nabla T_{c}^{(i)}\left(r_{j}=a_{j}\right) & \text { for } \theta_{j} \in\left[\alpha_{j 1}, \alpha_{j 2}\right]\end{cases} \\
& =\sum_{m=-\infty}^{\infty}\left[K_{M} \lambda_{m}^{\prime}\left(a_{j}\right)+\phi_{m}\left(a_{j}\right)\right] e^{i m \theta_{j}}, \\
i, j=1,2, i \neq j . &
\end{aligned}
$$

The right-hand term in (14) represents the Fourier expansion of a discontinuous function with different values at different angles. The new functions $\gamma_{m}\left(r_{j}\right)$ and $\phi_{m}\left(r_{j}\right)$ write (see Appendix):

$$
\begin{aligned}
\gamma_{m}\left(a_{j}\right)= & \frac{\beta_{j}}{\pi} \sum_{p=-\infty}^{\infty}\left[\eta_{p}^{(i)}\left(a_{j}\right)-\lambda_{p}\left(a_{j}\right)\right] e^{-i(m-p) \theta_{j i}} \\
& \times \frac{\sin \left\lfloor(m-p) \beta_{j}\right\rfloor}{(m-p) \beta_{j}}, \quad i, j=1,2, i \neq j \\
\phi_{m}\left(a_{j}\right)= & \frac{\beta_{j}}{\pi} \sum_{p=-\infty}^{\infty}\left[K_{c} \eta_{p}^{\prime(i)}\left(a_{j}\right)-K_{M} \lambda_{p}^{\prime}\left(a_{j}\right)\right] e^{-i(m-p) \theta_{j i}} \\
& \times \frac{\sin \left\lfloor(m-p) \beta_{j}\right\rfloor}{(m-p) \beta_{j}}, \quad i, j=1,2, i \neq j
\end{aligned}
$$

Here $\eta_{m}^{\prime}$ and $\lambda_{m}^{\prime}$ are the derivatives of the functions $\eta_{m}$ and $\lambda_{m}$.

Substituting (12) into (14), we obtain the following system of $4 m$ equations from which the $4 m$ constants $A_{m}^{(1)}$, $A_{m}^{(2)}, B_{m}^{(1)}$, and $B_{m}^{(2)}$ are obtained:

$\lambda_{m}\left(a_{j}\right)+\gamma_{m}\left(a_{j}\right)=B_{m}^{(j)} J_{m}\left(q_{c} a_{j}\right), \quad j=1,2$,

$K_{M} \lambda_{m}^{\prime}\left(a_{j}\right)+\phi_{m}\left(a_{j}\right)=K_{c} q_{c} B_{m}^{(j)} J_{m}^{\prime}\left(q_{c} a_{j}\right), \quad j=1,2$.

By comparing (10) and (16) it can be seen that the contribution of the overlapping between cylinders is accounted for by the expressions $\gamma_{m}\left(a_{j}\right)$ and $\phi_{m}\left(a_{j}\right)$ given by (15). It is worth noting that (16) reduce to (10) for nonoverlapping cylinders, since $\gamma_{m}\left(a_{j}\right)$ and $\phi_{m}\left(a_{j}\right)$ vanish as the overlapping semi-angle $\beta_{j}$ tends to zero.

As in the case of nonoverlapping cylinders, the method is also valid for a periodic line illumination parallel to the cylinders, provided that the incident plane thermal wave is changed by a cylindrical one, according to (11).

Moreover, by using the inverse Laplace transform [14], the modulated solutions we have obtained can be used to calculate the temperature evolution of the sample after being heated by a brief light pulse. This means that this theoretical approach can be used in both lock-in and pulsed photothermal techniques.

\section{Numerical calculations}

Equations (6) and (8) allow us to calculate the temperature at any point of the material, both for plane and line illumination. However, our calculations are restricted to the sample surface, where experimental measurements can be done. Moreover, calculations are performed for plane illumination, which is the most used in NDE. Before calculating the surface temperature for a given geometry, a test of convergence must be performed in order to determine the number of terms required in the summations of (6), (7a), and (7b). Our criterion is that the convergence is reached when the addition of another term to the series increases the surface temperature less than $0.1 \%$. It is worth noting that the minimum value of $m\left(m_{\min }\right)$ that guarantees the convergence increases as the cylinder approaches the surface $(d / a$ close to 1 ) and also as the degree of overlapping between cylinders increases. On the other hand, the convergence is almost independent of $\mu$. In fact, for a given value of $d / a, m_{\min }$ increases slightly as $\mu$ decreases. However, since thermal waves are highly damped, values of $\mu<d-a$ produce negligible changes in the surface temperature and therefore are out of interest.

In Fig. 6 calculations of the amplitude and phase of the surface temperature on a matrix with two equal cylinders $\left(a_{1}=a_{2}=0.5 \mathrm{~mm}\right)$ buried at the same depth $\left(\Delta_{1}=\right.$ $\left.d_{1}-a_{1}=\Delta_{2}=d_{2}-a_{2}=0.1 \mathrm{~mm}\right)$ are shown. The distance between cylinders is varied from $h=1.1 \mathrm{~mm}$ (nonoverlapping cylinders, continuous line), $h=1.0 \mathrm{~mm}$ (just touching cylinders, dotted line), and $h=0.9 \mathrm{~mm}$ (overlapping cylinders, dashed line). Two thermal diffusion lengths have been considered, $\mu_{M}=0.25 \mathrm{~mm}$ (thick lines) and $\mu_{M}=$ $1.5 \mathrm{~mm}$ (thin lines). On the left, calculations for isolating cylinders $\left(K_{c}=0\right)$ are shown, while on the right calculations for highly conducting cylinders $\left(\mu_{c}=10 \mu_{M}\right.$ and $\left.K_{c}=100 K_{M}\right)$ are drawn. In all the simulations the amplitude and phase of the surface temperature are normalized to the values obtained far away from the cylinders. As can be seen, the amplitude of the normalized surface temperature is enhanced on top of isolating cylinders, while it decreases above conducting cylinders. This behavior, which is independent of the thermal diffusion length, is due to the interference between incident and scattered thermal waves: constructive in the case of isolating cylinders and destructive in the case of conducting cylinders. On the other hand, the phase of the temperature shows the opposite trend with respect to the amplitude but only for short thermal diffusion lengths. Actually as the thermal diffusion length increases, the phase contrast changes from negative to positive for isolating cylinders and from positive to negative for conducting cylinders. It is worth noting that as the distance between cylinders is reduced, the resolution becomes poorer. 
Fig. 6 Normalized amplitude and phase of the surface temperature of a sample with two equal cylinders $\left(a_{1}=a_{2}=0.5 \mathrm{~mm}\right)$ buried at the same depth

$\left(\Delta_{1}=\Delta_{2}=0.1 \mathrm{~mm}\right)$. The distance between cylinders is varied from $h=1.1 \mathrm{~mm}$ (continuous line), $h=1.0 \mathrm{~mm}$ (dotted line), and $h=0.9 \mathrm{~mm}$ (dashed line). Two thermal diffusion lengths have been considered: $\mu_{M}=0.25 \mathrm{~mm}$ (thick lines) and $\mu_{M}=1.5 \mathrm{~mm}$ (thin lines). (a) Isolating cylinders $\left(K_{c}=0\right)$. (b) Highly conducting cylinders $\left(\mu_{c}=10 \mu_{M}\right.$ and $\left.K_{c}=100 K_{M}\right)$

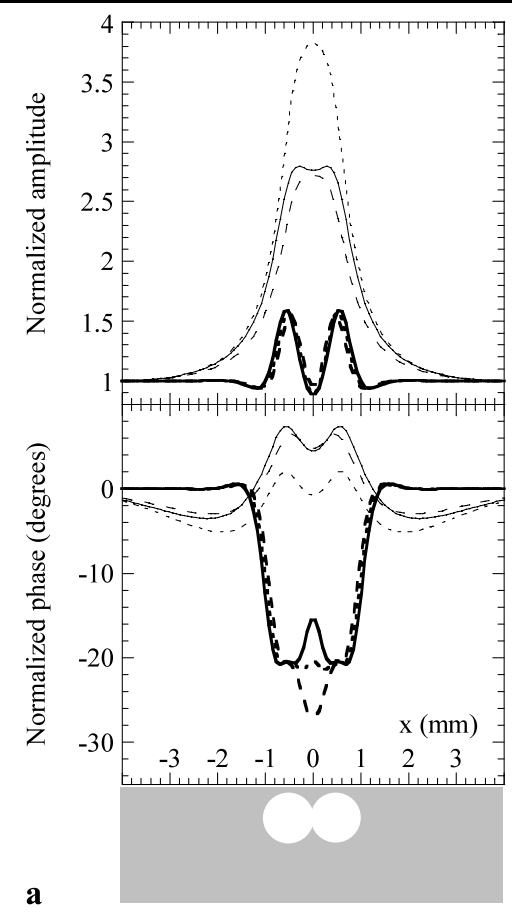

In particular for large thermal diffusion lengths both cylinders are hardly distinguished. However, the transition between nonoverlapping to overlapping cylinders is smooth, both in amplitude and phase. In this way, the absence of any singularity or jump just at the transition indicates the consistency of the theoretical model.

\section{Experimental results and conclusions}

To validate the model developed in Sect. 2.1 we have measured the surface temperature on calibrated samples using an infrared video camera. A multimode Nd:YAG laser is used to heat the sample surface. Its beam is defocused to a diameter of $5 \mathrm{~cm}$ in order to guarantee plane excitation, and modulated by means of a mechanical chopper. The IR signal emitted between 3.6 and $5 \mu \mathrm{m}$ is captured by an infrared camera (JADE J550M from CEDIP). By using a $50 \mathrm{~mm}$ lens together with two extension rings the minimum working distance is $7.5 \mathrm{~cm}$. Under these conditions, each pixel measures the average temperature over a square on the sample of $40 \mu \mathrm{m}$ in side. The lock-in software provides the amplitude and phase of the oscillating temperature. To improve the signal to noise ratio we record for each experiment 5000 images (170 frames per second during $30 \mathrm{~s}$ ). As the noise level is inversely proportional to the square root of the total number of images [15], we obtain a temperature noise level as low as $1 \mathrm{mK}$.

Calibrated samples have been prepared by drilling two cylindrical holes, each one with a radius of $0.5 \mathrm{~mm}$, in rigid graphite slabs (purity $99.95 \%$ and $D=75 \mathrm{~mm}^{2} / \mathrm{s}$ ). Several samples with overlapping and nonoverlapping cylinders have been prepared. The free surface has been sandpapered to reduce the depth of the hole beneath the surface $(\Delta=d-a)$.

In Fig. 7(a) the normalized amplitude and phase of the surface temperature of a sample with two nonoverlapping buried cylinders are shown by dots. The geometrical data were measured by an optical microscope: $\Delta_{1}=110 \mu \mathrm{m}$, $\Delta_{2}=155 \mu \mathrm{m}$, and $h=1.30 \mathrm{~mm}$. The same measurements for a sample containing two overlapping subsurface cylinders are shown in Fig. 7(b). In this sample the geometrical data are: $\Delta_{1}=112 \mu \mathrm{m}, \Delta_{2}=152 \mu \mathrm{m}$, and $h=0.79 \mathrm{~mm}$. Measurements were performed at various frequencies in the range from $40 \mathrm{~Hz}$ to $950 \mathrm{~Hz}$. Here the results for $80 \mathrm{~Hz}$ and $190 \mathrm{~Hz}$ are shown. The continuous lines correspond to the numerical calculations using (6) with the thermal and geometrical parameters specified above. As can be seen, the agreement between the experimental measurements and the numerical calculations is very good, validating the theoretical model developed in Sect. 2.1 for overlapping cylinders.

In this paper we have studied the multiple scattering of thermal waves by two overlapping subsurface cylinders. The main difference with respect to the already-known solution for nonoverlapping cylinders is the modification of the boundary conditions. Measurements performed on calibrated samples using infrared thermography indicate the validity of the model. This paper should be understood as the key-work to study the heat conduction in composite materials filled with interconnected inclusions. This is the case of inverse opals as well as fiber reinforced composites filled 
Fig. 7 (a) Normalized amplitude and phase of the surface temperature of a graphite sample with two nonoverlapping cylindrical holes $\left(a_{1}=a_{2}=0.5 \mathrm{~mm}\right.$, $\Delta_{1}=110 \mu \mathrm{m}, \Delta_{2}=155 \mu \mathrm{m}$, and $h=1.30 \mathrm{~mm}$ ). (b) The same for two overlapping cylindrical holes $\left(a_{1}=a_{2}=0.5\right.$ $\mathrm{mm}, \Delta_{1}=112 \mu \mathrm{m}$, $\Delta_{2}=152 \mu \mathrm{m}$, and $h=0.79 \mathrm{~mm}$ ). Measurements for $80 \mathrm{~Hz}$ and $190 \mathrm{~Hz}$ are shown. Dots and crosses are the experimental results, while the continuous lines correspond to the numerical calculations

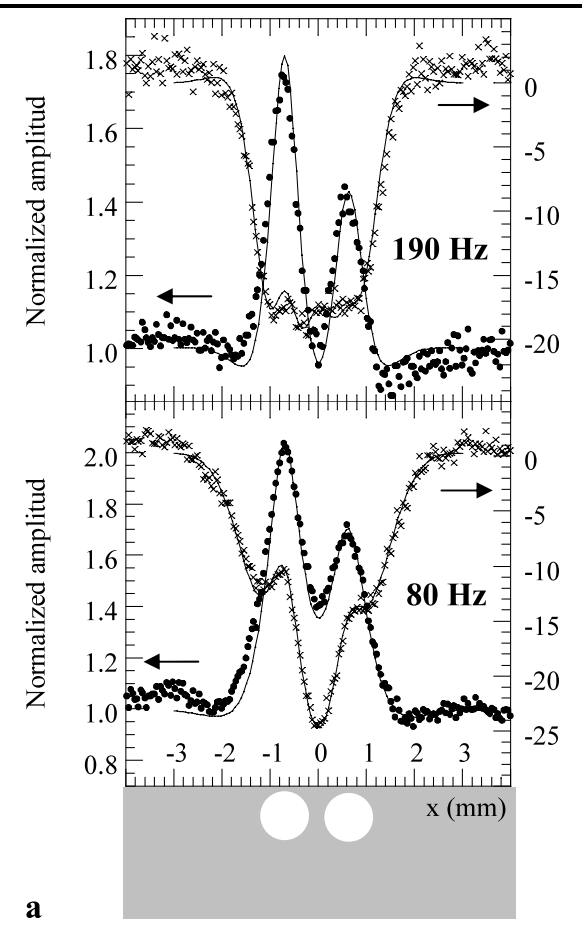

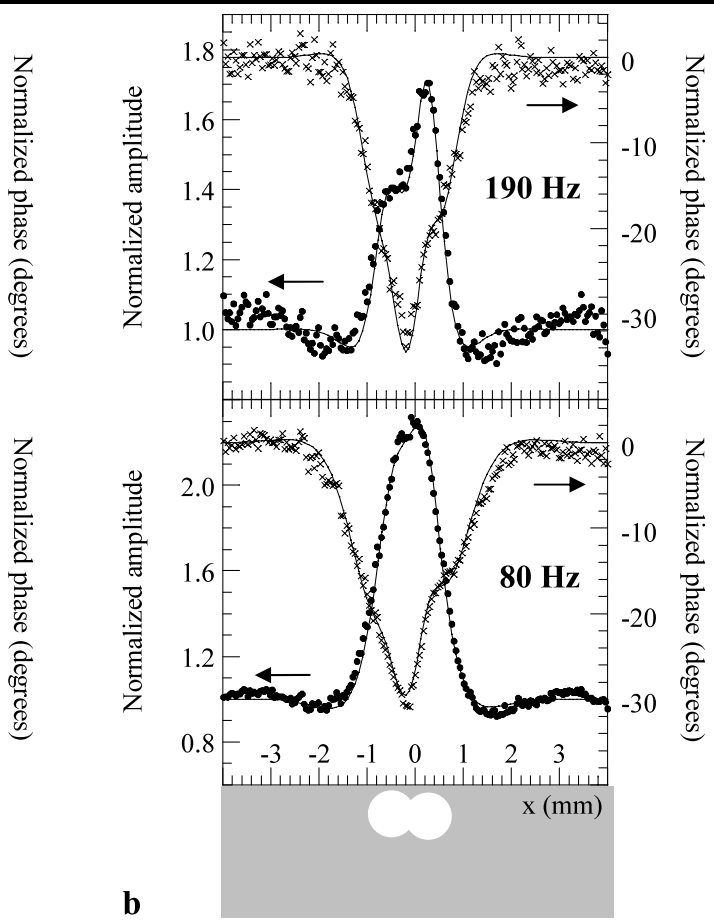

with a high fiber's volume fraction, for which anomalous high values of the effective thermal conductivity have been found.

Acknowledgements This work has been supported by the Ministerio de Educación y Ciencia (MAT2005-02999), by the Universidad del País Vasco (DIPE06/06) and by the Diputación General de Aragón. Messias Costa acknowledges a predoctoral fellowship from Coordenação de Aperfeiçoamento de Pessoal de Nível Superior-Ministério da Educação.

\section{Appendix: Fourier expansion of the boundary conditions}

Theorem 1 The Fourier expansion corresponding to the temperature continuity given by (14a) can be expressed as:

$$
\begin{aligned}
& \left.\begin{array}{l}
T_{M}\left(r_{j}=a_{j}\right) \quad \text { for } \theta_{j} \notin\left[\alpha_{j 1}, \alpha_{j 2}\right] \\
T_{c}^{(i)}\left(r_{j}=a_{j}\right) \quad \text { for } \theta_{j} \in\left[\alpha_{j 1}, \alpha_{j 2}\right]
\end{array}\right\} \\
& \quad=\sum_{m=-\infty}^{\infty}\left[\lambda_{m}\left(a_{j}\right)+\gamma_{m}\left(a_{j}\right)\right] e^{i m \theta_{j}}, \quad i, j=1,2, i \neq j,
\end{aligned}
$$

where

$$
\begin{aligned}
\gamma_{m}\left(a_{j}\right)= & \frac{\beta_{j}}{\pi} \sum_{p=-\infty}^{\infty}\left[\eta_{p}^{(i)}\left(a_{j}\right)-\lambda_{p}\left(a_{j}\right)\right] e^{-i(m-p) \theta_{j i}} \\
& \times \frac{\sin \left\lfloor(m-p) \beta_{j}\right\rfloor}{(m-p) \beta_{j}}, \quad i, j=1,2, i \neq j .
\end{aligned}
$$

Proof According to the Fourier expansion, the coefficients $\lambda_{m}\left(r_{j}\right), \eta_{m}^{(i)}\left(a_{j}\right)$, and $\gamma_{m}\left(r_{j}\right)$ defined by (6), (12), and (14) can be expressed in integral form as

$$
\begin{aligned}
& \lambda_{m}\left(a_{j}\right)=\frac{1}{2 \pi} \int_{0}^{2 \pi} T_{M}\left(a_{j}, \theta_{j}\right) e^{-i m \theta_{j}} d \theta_{j}, \\
& \eta_{m}^{(i)}\left(a_{j}\right)=\frac{1}{2 \pi} \int_{0}^{2 \pi} T_{c}^{(i)}\left(a_{j}, \theta_{j}\right) e^{-i m \theta_{j}} d \theta_{j}, \\
& \lambda_{m}\left(a_{j}\right)+\gamma_{m}\left(a_{j}\right) \\
& =\frac{1}{2 \pi} \int_{\left[0, \alpha_{j 1}\right] \cup\left[\alpha_{j 2}, 2 \pi\right]} T_{M}\left(a_{j}, \theta_{j}\right) e^{-i m \theta_{j}} d \theta_{j} \\
& \quad+\frac{1}{2 \pi} \int_{\alpha_{j 1}}^{\alpha_{j 2}} T_{c}^{(i)}\left(a_{j}, \theta_{j}\right) e^{-i m \theta_{j}} d \theta_{j} \\
& =\frac{1}{2 \pi} \int_{0}^{2 \pi} T_{M}\left(a_{j}, \theta_{j}\right) e^{-i m \theta_{j}} d \theta_{j} \\
& \quad-\frac{1}{2 \pi} \int_{\alpha_{j 1}}^{\alpha_{j 2}} T_{M}\left(a_{j}, \theta_{j}\right) e^{-i m \theta_{j}} d \theta_{j} \\
& \quad+\frac{1}{2 \pi} \int_{\alpha_{j 1}}^{\alpha_{j 2}} T_{c}^{(i)}\left(a_{j}, \theta_{j}\right) e^{-i m \theta_{j}} d \theta_{j} .
\end{aligned}
$$

According to (19), the first term in last expression is $\lambda_{m}\left(a_{j}\right)$. Therefore $\gamma_{m}\left(a_{j}\right)$ is given by

$$
\begin{aligned}
\gamma_{m}\left(a_{j}\right)= & -\frac{1}{2 \pi} \int_{\alpha_{j 1}}^{\alpha_{j 2}} T_{M}\left(a_{j}, \theta_{j}\right) e^{-i m \theta_{j}} d \theta_{j} \\
& +\frac{1}{2 \pi} \int_{\alpha_{j 1}}^{\alpha_{j 2}} T_{c}^{(i)}\left(a_{j}, \theta_{j}\right) e^{-i m \theta_{j}} d \theta_{j}
\end{aligned}
$$




$$
\begin{aligned}
= & -\frac{1}{2 \pi} \int_{\alpha_{j 1}}^{\alpha_{j 2}}\left(\sum_{p=-\infty}^{\infty} \lambda_{p}\left(a_{j}\right) e^{i p \theta_{j}}\right) e^{-i m \theta_{j}} d \theta_{j} \\
& +\frac{1}{2 \pi} \int_{\alpha_{j 1}}^{\alpha_{j 2}}\left(\sum_{p=-\infty}^{\infty} \eta_{p}^{(i)}\left(a_{j}\right) e^{i p \theta_{j}}\right) e^{-i m \theta_{j}} d \theta_{j} .
\end{aligned}
$$

The solution of the integral is given by

$$
\begin{aligned}
\int_{a}^{b} & \left(\sum_{p=-\infty}^{\infty} \xi_{p}\left(a_{j}\right) e^{i p \theta}\right) e^{-i m \theta} d \theta \\
= & (b-a) \xi_{m}\left(a_{j}\right) \\
& +\sum_{\substack{p=-\infty \\
p \neq m}}^{\infty} \xi_{p}\left(a_{j}\right) \frac{e^{-i(m-p) b}-e^{-i(m-p) a}}{i(p-m)} .
\end{aligned}
$$

Therefore (22) is written as

$$
\begin{aligned}
\gamma_{m}\left(a_{j}\right)= & -\frac{1}{2 \pi}\left[2 \beta_{j} \lambda_{m}\left(a_{j}\right)\right. \\
& \left.+\sum_{\substack{p=-\infty \\
p \neq m}}^{\infty} \lambda_{p}\left(a_{j}\right) \frac{e^{-i(m-p) \alpha_{j 2}}-e^{-i(m-p) \alpha_{j 1}}}{i(p-m)}\right] \\
& +\frac{1}{2 \pi}\left[2 \beta_{j} \eta_{m}^{(i)}\left(a_{j}\right)\right. \\
& \left.+\sum_{\substack{p=-\infty \\
p \neq m}}^{\infty} \eta_{p}^{(i)}\left(a_{j}\right) \frac{e^{-i(m-p) \alpha_{j 2}}-e^{-i(m-p) \alpha_{j 1}}}{i(p-m)}\right] .
\end{aligned}
$$

According to the geometry shown in Fig. 5, $\alpha_{j 1}=\theta_{j i}-$ $\beta_{j}$ and $\alpha_{j 2}=\theta_{j i}+\beta_{j}$. Therefore the exponentials in (24) can be simplified as

$$
\begin{aligned}
& \frac{e^{-i(m-p) \alpha_{j 2}}-e^{-i(m-p) \alpha_{j 2}}}{2 i} \\
& =\frac{e^{-i(m-p)\left(\theta_{j i}+\beta_{j}\right)}-e^{-i(m-p)\left(\theta_{j i}+\beta_{j}\right)}}{2 i} \\
& =e^{-i(m-p) \theta_{j i}\left[\frac{e^{-i(m-p) \beta_{j}}-e^{i(m-p) \beta_{j}}}{2 i}\right]} \\
& =-e^{-i(m-p) \theta_{j i}} \sin \left[(m-p) \beta_{j}\right] .
\end{aligned}
$$

Finally, substituting (25) into (24), we obtain

$$
\begin{aligned}
\gamma_{m}\left(a_{j}\right)= & \frac{\beta_{j}}{\pi}\left[\eta_{m}^{(i)}\left(a_{j}\right)-\lambda_{m}\left(a_{j}\right)\right] \\
& -\sum_{\substack{p=-\infty \\
p \neq m}}^{\infty} \frac{\eta_{p}^{(i)}\left(a_{j}\right)-\lambda_{p}\left(a_{j}\right)}{\pi(p-m)} e^{-i(m-p) \theta_{j i}}
\end{aligned}
$$

$$
\begin{aligned}
& \times \sin \left[(m-p) \beta_{j}\right] \\
= & \frac{\beta_{j}}{\pi}\left[\eta_{m}^{(i)}\left(a_{j}\right)-\lambda_{m}\left(a_{j}\right)\right] \\
& +\frac{\beta_{j}}{\pi} \sum_{\substack{p=-\infty \\
p \neq m}}^{\infty}\left[\eta_{p}^{(i)}\left(a_{j}\right)-\lambda_{p}\left(a_{j}\right)\right] e^{-i(m-p) \theta_{j i}} \\
& \times \frac{\sin \left\lfloor(m-p) \beta_{j}\right\rfloor}{(m-p) \beta_{j}} \\
= & \frac{\beta_{j}}{\pi} \sum_{p=-\infty}^{\infty}\left[\eta_{p}^{(i)}\left(a_{j}\right)-\lambda_{p}\left(a_{j}\right)\right] e^{-i(m-p) \theta_{j i}} \\
& \times \frac{\sin \left\lfloor(m-p) \beta_{j}\right\rfloor}{(m-p) \beta_{j}} .
\end{aligned}
$$

Theorem 2 The Fourier expansion corresponding to the heat flow continuity given by (14a) and (14b) can be expressed as:

$$
\begin{aligned}
& K_{M} \nabla T_{M}\left(r_{j}=a_{j}\right) \quad \text { for } \theta_{j} \notin\left[\alpha_{j 1}, \alpha_{j 2}\right] \\
& \left.K_{c} \nabla T_{c}^{(2)}\left(r_{j}=a_{j}\right) \quad \text { for } \theta_{j} \in\left[\alpha_{j 1}, \alpha_{j 2}\right]\right\} \\
& =\sum_{m=-\infty}^{\infty}\left[K_{M} \lambda_{m}^{\prime}\left(a_{j}\right)+\phi_{m}\left(a_{j}\right)\right] e^{i m \theta_{j}}, \\
& i, j=1,2, i \neq j
\end{aligned}
$$

where

$$
\begin{aligned}
\phi_{m}\left(a_{j}\right)= & \frac{\beta_{j}}{\pi} \sum_{p=-\infty}^{\infty}\left[K_{c} \eta_{p}^{\prime(i)}\left(a_{j}\right)-K_{M} \lambda_{p}^{\prime}\left(a_{j}\right)\right] e^{-i(m-p) \theta_{j i}} \\
& \times \frac{\sin \left\lfloor(m-p) \beta_{j}\right\rfloor}{(m-p) \beta_{j}}, \quad i, j=1,2, i \neq j,
\end{aligned}
$$

$\eta_{m}^{\prime}$ and $\lambda_{m}^{\prime}$ being the derivatives of the functions $\eta_{m}$ and $\lambda_{m}$.

Proof The radial derivatives of (19) and (20) are given by

$$
\begin{aligned}
& \lambda_{m}^{\prime}\left(a_{j}\right)=\frac{1}{2 \pi} \int_{0}^{2 \pi} T_{M}^{\prime}\left(a_{j}, \theta_{j}\right) e^{-i m \theta_{j}} d \theta_{j}, \\
& \eta_{m}^{\prime(i)}\left(a_{j}\right)=\frac{1}{2 \pi} \int_{0}^{2 \pi} T_{c}^{\prime(i)}\left(a_{j}, \theta_{j}\right) e^{-i m \theta_{j}} d \theta_{j} .
\end{aligned}
$$

On the other hand, according to the Fourier expansion, the coefficients $\phi_{m}\left(r_{j}\right)$, which are defined by (14), can be expressed in integral form as

$$
\begin{aligned}
& K_{M} \lambda_{m}^{\prime}\left(a_{j}\right)+\phi_{m}\left(a_{j}\right) \\
& \quad=\frac{1}{2 \pi} \int_{\left[0, \alpha_{j 1}\right] \cup\left[\alpha_{j 2}, 2 \pi\right]} K_{M} T_{M}^{\prime}\left(a_{j}, \theta_{j}\right) e^{-i m \theta_{j}} d \theta_{j}
\end{aligned}
$$




$$
\begin{aligned}
& +\frac{1}{2 \pi} \int_{\alpha_{j 1}}^{\alpha_{j 2}} K_{c} T_{c}^{\prime(i)}\left(a_{j}, \theta_{j}\right) e^{-i m \theta_{j}} d \theta_{j} \\
= & \frac{1}{2 \pi} \int_{0}^{2 \pi} K_{M} T_{M}^{\prime}\left(a_{j}, \theta_{j}\right) e^{-i m \theta_{j}} d \theta_{j} \\
& -\frac{1}{2 \pi} \int_{\alpha_{j 1}}^{\alpha_{j 2}} K_{M} T_{M}^{\prime}\left(a_{j}, \theta_{j}\right) e^{-i m \theta_{j}} d \theta_{j} \\
& +\frac{1}{2 \pi} \int_{\alpha_{j 1}}^{\alpha_{j 2}} K_{c} T_{c}^{\prime(i)}\left(a_{j}, \theta_{j}\right) e^{-i m \theta_{j}} d \theta_{j} .
\end{aligned}
$$

According to (29), the first term in last expression is $K_{M} \lambda_{m}^{\prime}\left(r_{j}\right)$. Therefore $\phi_{m}\left(r_{j}\right)$ is given by

$$
\begin{aligned}
\phi_{m}\left(a_{j}\right)= & -\frac{1}{2 \pi} \int_{\alpha_{j 1}}^{\alpha_{j 2}} K_{M} T_{M}^{\prime}\left(a_{j}, \theta_{j}\right) e^{-i m \theta_{j}} d \theta_{j} \\
& +\frac{1}{2 \pi} \int_{\alpha_{j 1}}^{\alpha_{j 2}} K_{c} T_{c}^{\prime(i)}\left(a_{j}, \theta_{j}\right) e^{-i m \theta_{j}} d \theta_{j} \\
= & -\frac{1}{2 \pi} \int_{\alpha_{j 1}}^{\alpha_{j 2}} K_{M}\left(\sum_{p=-\infty}^{\infty} \lambda_{p}^{\prime}\left(a_{j}\right) e^{i p \theta_{j}}\right) e^{-i m \theta_{j}} d \theta_{j} \\
& +\frac{1}{2 \pi} \int_{\alpha_{j 1}}^{\alpha_{j 2}} K_{c}\left(\sum_{p=-\infty}^{\infty} \eta_{p}^{\prime(i)}\left(a_{j}\right) e^{i p \theta_{j}}\right) \\
& \times e^{-i m \theta_{j}} d \theta_{j} .
\end{aligned}
$$

Taking into account (23), (32) can be written as

$$
\begin{aligned}
\phi_{m}\left(a_{j}\right)= & -\frac{1}{2 \pi}\left[2 \beta_{j} K_{M} \lambda_{m}^{\prime}\left(a_{j}\right)\right. \\
& \left.+\sum_{\substack{p=-\infty \\
p \neq m}}^{\infty} K_{M} \lambda_{p}^{\prime}\left(a_{j}\right) \frac{e^{-i(m-p) \alpha_{j 2}-e^{-i(m-p) \alpha_{j 1}}}}{i(p-m)}\right] \\
& +\frac{1}{2 \pi}\left[2 \beta_{j} K_{c} \eta_{m}^{\prime(i)}\left(a_{j}\right)\right. \\
& +\sum_{\substack{p=-\infty \\
p \neq m}}^{\infty} K_{c} \eta_{p}^{\prime(i)}\left(a_{j}\right) \\
& \left.\times \frac{e^{-i(m-p) \alpha_{j 2}-e^{-i(m-p) \alpha_{j 1}}}}{i(p-m)}\right] .
\end{aligned}
$$

Finally, substituting (25) into (33), we obtain

$$
\phi_{m}\left(a_{j}\right)=\frac{\beta_{j}}{\pi}\left[K_{c} \eta_{m}^{\prime(i)}\left(a_{j}\right)-K_{M} \lambda_{m}^{\prime}\left(a_{j}\right)\right]
$$

$$
\begin{aligned}
& -\sum_{\substack{p=-\infty \\
p \neq m}}^{\infty} \frac{K_{c} \eta_{p}^{(i)}\left(a_{j}\right)-K_{M} \lambda_{p}^{\prime}\left(a_{j}\right)}{\pi(p-m)} e^{-i(m-p) \theta_{j i}} \\
& \times \sin \left[(m-p) \beta_{j}\right] \\
= & \frac{\beta_{j}}{\pi}\left[K_{c} \eta_{m}^{\prime(i)}\left(a_{j}\right)-K_{M} \lambda_{m}^{\prime}\left(a_{j}\right)\right] \\
& +\frac{\beta_{j}}{\pi} \sum_{\substack{p=-\infty \\
p \neq m}}^{\infty}\left[K_{c} \eta_{p}^{\prime(i)}\left(a_{j}\right)-K_{M} \lambda_{p}^{\prime}\left(a_{j}\right)\right] \\
& \times e^{-i(m-p) \theta_{j i}} \frac{\sin \left\lfloor(m-p) \beta_{j}\right\rfloor}{(m-p) \beta_{j}} \\
= & \frac{\beta_{j}}{\pi} \sum_{p=-\infty}^{\infty}\left[K_{c} \eta_{p}^{\prime(i)}\left(a_{j}\right)-K_{M} \lambda_{p}^{\prime}\left(a_{j}\right)\right] e^{-i(m-p) \theta_{j i}} \\
& \times \frac{\sin \left\lfloor(m-p) \beta_{j}\right\rfloor}{(m-p) \beta_{j}} .
\end{aligned}
$$

\section{References}

1. D.P. Almond, P.M. Patel, Photothermal Science and Techniques (Chapman and Hall, London, 1996)

2. N.C. Fernelius, J. Appl. Phys. 51, 650 (1980)

3. C.A. Bennet, R.R. Patty, Appl. Opt. 21, 49 (1982)

4. J.M. Terrón, A. Salazar, A. Sánchez-Lavega, J. Appl. Phys. 91, 1087 (2002)

5. M.L. Rapún, F.J. Sayas, Arch. Comput. Methods Eng. 14, 3 (2007)

6. F. Garrido, A. Salazar, J. Appl. Phys. 95, 140 (2004)

7. A.E. Aliev, S.B. Lee, R.H. Baughman, A.A. Zakhidov, J. Luminescence 125, 11 (2007)

8. F.J. Macedo, private communication

9. G.B. Arfken, H.J. Weber, Mathematical Methods for Physicists, 6th edn. (Academic, New York, 2005), p. 687

10. P.M. Morse, K.U. Ingard, Theoretical Acoustics (McGraw-Hill, New York, 1968), p. 401

11. G.N. Watson, A Treatise on the Theory of Bessel Functions (Cambridge University, London, 1966), pp. 359-361

12. P.M. Morse, H. Feshbach, Methods of Theoretical Physics (McGraw-Hill, New York, 1953), p. 1372

13. A. Salazar, A. Sánchez-Lavega, R. Celorrio, J. Appl. Phys. 93, 4536 (2003)

14. D. Maillet, S. André, J.C. Batsale, A. Degiovanni, C. Moyne, Thermal Quadrupoles (Wiley, New York, 2000), pp. 333-355

15. O. Breitenstein, M. Langenkamp, Lock-in Thermography (Springer, Berlin, 2003), p. 32 